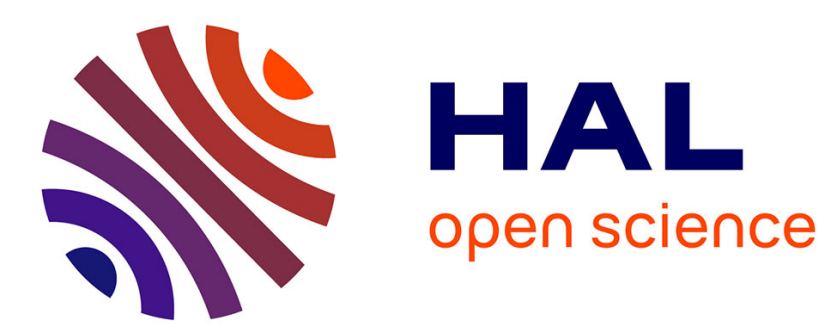

\title{
Value Function of an Infinite Dimensional Infinite Horizon Problem
}

Hélène Frankowska, Nobusumi Sagara

\section{To cite this version:}

Hélène Frankowska, Nobusumi Sagara. Value Function of an Infinite Dimensional Infinite Horizon Problem. 2020 European Control Conference (ECC), May 2020, Saint Petersburg, Russia. hal02906906

\section{HAL Id: hal-02906906 https://hal.science/hal-02906906}

Submitted on 26 Jul 2020

HAL is a multi-disciplinary open access archive for the deposit and dissemination of scientific research documents, whether they are published or not. The documents may come from teaching and research institutions in France or abroad, or from public or private research centers.
L'archive ouverte pluridisciplinaire HAL, est destinée au dépôt et à la diffusion de documents scientifiques de niveau recherche, publiés ou non, émanant des établissements d'enseignement et de recherche français ou étrangers, des laboratoires publics ou privés. 


\title{
Value Function of an Infinite Dimensional Infinite Horizon Problem
}

\author{
Helene Frankowska ${ }^{1}$ and Nobusumi Sagara ${ }^{2}$
}

\begin{abstract}
We investigate the value function of an infinite horizon problem in the setting of an infinite-dimensional differential inclusion. In particular, we provide an upper estimate of its Gateaux subdifferential in terms of the Clarke subdifferential of the integrand and the Clarke normal cone to the graph of the set-valued dynamics. We also derive a necessary optimality condition in the form of an Euler-Lagrange inclusion, the maximum principle and a sensitivity relation.
\end{abstract}

\section{INTRODUCTION}

Optimal control and dynamic programming are instrumental cornerstones of modern economic growth theory originated in [28]. In the general reduced model of capital accumulation, necessary (and sufficient) conditions for optimality are employed under the convexity assumptions on utility functions and technologies to investigate the existence of competitive equilibria and support prices [7], [27], [34], transversality conditions at infinity [1], [7], and the uniqueness and global stability of stationary states [11], [14], [30], [31]. One of the advantages of convex economic models lies in the crucial observation that the differentiability of the value function is guaranteed under the smoothness assumptions on data, see [6], [7], [9], [29].

On the contrary, the absence of convexity and smoothness are two major sources of complex economic dynamics in continuous time as illustrated in [2], [8], [20], [25], [33], [35]. The lack of convexity results in the failure of the differentiability of the value function even if the underlying data are smooth. One can expect at best the local Lipschitz continuity of the value function. This causes problems with expressing optimal synthesis in many nonconvex economic growth models when one attempts to apply the HamiltonJacobi-Bellman (HJB) theory.

The failure of differentiability of the value function has stimulated two alternative approaches. One is the application of a "generalized" subdifferential calculus along the lines of [15], leading to the formulation of a relation between the maximum principle and the value function [16], [17]. The other one is the concept of "viscosity solutions" to the associated HJB equation (initiated in [18], [19]), whose unique (viscosity) solution is the value function. For the connections between the maximum principle and the superdifferentials of the value function, see also [13], [21], [23].

\footnotetext{
*Research of the first author benefited from the support of the FJMH Program PGMO and from the support to this program from EDF-THALESORANGE-CRITEO. Research of the second author benefited from the support of the JSPS KAKENHI grant 26380246 from the Ministry of Education, Culture, Sports, Science and Technology, Japan.

${ }^{1}$ Frankowska H. is with CNRS, IMJ-PRG, Sorbonne Université, Paris, France helene.frankowska@imj-prg.fr

${ }^{2}$ Sagara N. is with Department of Economics, Hosei University, Tokyo, Japan nsagara@hosei.ac.jp
}

The purpose of this paper is twofold. Firstly, we provide an upper estimate of the Gateaux subdifferential of the value function in terms of the Clarke subdifferential and the Clarke normal cone to the set-valued mapping describing dynamics.

Secondly, we derive a necessary condition for optimality in the form of an adjoint inclusion, the maximum principle and a relation of the adjoint variable to the subdifferentials of the value function along the optimal trajectory. To deal with the adjoint variable in dual spaces, we use the Gelfand integrals of the Gateaux and Clarke subdifferential mappings, which is a new feature that does not arise in the context of finitedimensional control systems. We also consider the relaxed variational problem, which is a suitable convexification of the original variational problem, and derive the necessary condition that narrows the class of candidates for optimality. Our dynamical system in the form of a differential inclusion being very general, these results are derived under quite restrictive "interiority assumption" $\left(H_{8}\right)$. In the particular case of dynamics described by a control system we may remove assumption $\left(H_{8}\right)$.

For the finite-dimensional infinite horizon problem, necessary conditions without convexity assumptions using Gateaux, Clarke, and limiting subdifferentials were derived in [3], [13], [32], [36], while for the control systems in Hilbert spaces, a necessary condition under the convexity assumption was obtained in [5]. For the semilinear control systems in Banach spaces, the necessary and sufficient conditions were derived in [12] in the finite horizon setting when the sets of admissible velocities are convex.

The outline of the paper is as follows. Section 2 collects some definitions. In Section 3 we formulate the nonconvex variational problem and investigate the Lipschitz continuity and subdifferentiability of the value function. We derive in Sections 4, 5, 6 some necessary optimality conditions for the original and relaxed variational problems. Because of the lack of space some of proofs are omitted. They will be published elsewhere.

\section{PRELIMINARIES}

Let $(E,\|\cdot\|)$ be a real separable Banach space with the dual system $\left\langle E^{*}, E\right\rangle$, where $E^{*}$ is the norm dual of $E$. For a nonempty subset $C \subset E$ denote by $d_{C}: E \rightarrow \mathbb{R}_{+}$the distance function from the set $C$, by $K_{C}(x)$ and $T_{C}(x)$ respectively the contingent and Clarke tangent cones to $C$ at $x \in C$ and let $N_{C}(x)$ stand for the Clarke normal cone to $C$ at $x \in C$, see for instance [4, Chapter 4] or [15] for the precise definitions. The lower (resp. upper) directional derivative of $\varphi: E \rightarrow \mathbb{R} \cup\{+\infty\}$ at $\bar{x} \in \operatorname{dom} \varphi$ in the 
direction $v \in E$ is defined by

$$
\begin{aligned}
& \varphi^{-}(\bar{x} ; v):=\liminf _{\substack{u \rightarrow v \\
\theta \downarrow 0}} \frac{\varphi(\bar{x}+\theta u)-\varphi(\bar{x})}{\theta} \in \mathbb{R} \cup\{ \pm \infty\}, \\
& \varphi^{+}(\bar{x} ; v):=\limsup _{\substack{u \rightarrow v \\
\theta \downarrow 0}} \frac{\varphi(\bar{x}+\theta u)-\varphi(\bar{x})}{\theta} \in \mathbb{R} \cup\{ \pm \infty\} .
\end{aligned}
$$

respectively. The Dini-Hadamard subdifferential of $\varphi$ at $\bar{x}$ is

$$
\partial^{-} \varphi(\bar{x}):=\left\{x^{*} \in E^{*} \mid\left\langle x^{*}, v\right\rangle \leq \varphi^{-}(\bar{x} ; v) \forall v \in E\right\}
$$

and the Dini-Hadamard superdifferential of $\varphi$ at $\bar{x}$ is defined by

$$
\partial^{+} \varphi(\bar{x}):=\left\{x^{*} \in E^{*} \mid\left\langle x^{*}, v\right\rangle \geq \varphi^{+}(\bar{x} ; v) \forall v \in E\right\} .
$$

If $\varphi$ is locally Lipschitz at $\bar{x}$, denote by $\varphi^{\circ}(\bar{x} ; v)$ the Clarke directional derivative of $\varphi$ at $\bar{x}$ in the direction $v$, see [15] for the definition.

We denote by $\mathbb{R}_{+}$the set of all nonnegative reals. Below, by measurable, we shall always mean Lebesgue measurable. Let $W_{l o c}^{1,1}([t, \infty) ; E)$, where $t \geq 0$, denote the Sobolev space of continuous functions $x:[t, \infty) \rightarrow E$, whose derivative $\dot{x}(s)$ exists for a.e. $s \geq t, \dot{x}(\cdot)$ is locally Bochner integrable on $[t, \infty)$ and $x(\tau)=\int_{t}^{\tau} \dot{x}(s) d s+x(t)$ for every $\tau \in[t, \infty)$.

\section{VAlue Function}

Let $L: \mathbb{R}_{+} \times E \times E \rightarrow \mathbb{R} \cup\{+\infty\}$ be an integrand. Given an arc $x(\cdot) \in W_{\text {loc }}^{1,1}([t, \infty), E)$, define

$$
\int_{t}^{\infty} L(s, x(s), \dot{x}(s)) d s=\lim _{T \rightarrow \infty} \int_{t}^{T} L(s, x(s), \dot{x}(s)) d s
$$

for every $t \in \mathbb{R}_{+}$provided the above limit does exist.

Let $\Gamma: \mathbb{R}_{+} \times E \rightsquigarrow E$ be a multifunction. Define the set of admissible trajectories starting at time $t \in \mathbb{R}_{+}$from a given initial condition $\xi \in E$ by $\mathcal{A}_{(t, \xi)}=$

$\left\{x(\cdot) \in W_{\text {loc }}^{1,1}([t, \infty), E) \mid \dot{x}(s) \in \Gamma(s, x(s))\right.$ a.e., $\left.x(t)=\xi\right\}$.

Then the value function $V: \mathbb{R}_{+} \times E \rightarrow \mathbb{R} \cup\{ \pm \infty\}$ is

$$
V(t, \xi)=\inf _{x(\cdot) \in \mathcal{A}_{(t, \xi)}} \int_{t}^{\infty} L(s, x(s), \dot{x}(s)) d s .
$$

In the above we set $V(t, \xi)=+\infty$ if $\mathcal{A}_{(t, \xi)}$ is empty or if for every $x(\cdot) \in \mathcal{A}_{(t, \xi)}$ the integral $\int_{t}^{\infty} L(s, x(s), \dot{x}(s)) d s$ is not well-defined. The effective domain of $V$ is $\operatorname{dom} V=$ $\left\{(t, x) \in \mathbb{R}_{+} \times E \mid V(t, x)<+\infty\right\}$. For every $(t, \xi) \in$ dom $V$, an admissible trajectory $x(\cdot) \in \mathcal{A}_{(t, \xi)}$ is said to be optimal for $\left(P_{t}\right)$ if $\int_{t}^{\infty} L(s, x(s), \dot{x}(s)) d s=V(t, \xi)>-\infty$. The standing hypothesis are as follows.

$\left(\mathbf{H}_{\mathbf{1}}\right) \mathcal{A}_{(t, \xi)}$ is nonempty for every $(t, \xi) \in \mathbb{R}_{+} \times E$.

$\left(\mathbf{H}_{\mathbf{2}}\right) \quad L(\cdot, x, y)$ is measurable for every $(x, y) \in E \times E$.

$\left(\mathbf{H}_{3}\right)$ There exist integrable functions $l_{0}, l_{1}: \mathbb{R}_{+} \rightarrow$ $\mathbb{R}_{+}$, and a locally bounded, integrable function $l_{2}: \mathbb{R}_{+} \rightarrow \mathbb{R}_{+}$such that $|L(t, 0,0)| \leq l_{0}(t)$,

$$
\left|L(t, x, y)-L\left(t, x^{\prime}, y^{\prime}\right)\right| \leq l_{1}(t)\left\|x-x^{\prime}\right\|+l_{2}(t)\left\|y-y^{\prime}\right\|
$$

for a.e. $t \in \mathbb{R}_{+}$and all $(x, y),\left(x^{\prime}, y^{\prime}\right) \in E \times E$.

$\left(\mathbf{H}_{4}\right) \Gamma$ has nonempty closed values.
$\left(\mathbf{H}_{\mathbf{5}}\right) \Gamma(\cdot, x)$ is measurable for every $x \in E$.

$\left(\mathbf{H}_{6}\right)$ There exist locally integrable $\gamma_{0}: \mathbb{R}_{+} \rightarrow \mathbb{R}_{+}$and $\gamma_{1}: \mathbb{R}_{+} \rightarrow \mathbb{R}_{+}$such that $\Gamma(t, 0) \subset \gamma_{0}(t) B$,

$$
\Gamma(t, x) \subset \Gamma\left(t, x^{\prime}\right)+\gamma_{1}(t)\left\|x-x^{\prime}\right\| B
$$

for a.e. $t \in \mathbb{R}_{+}$and all $x, x^{\prime} \in E$.

$\left(\mathbf{H}_{7}\right)$

$$
\begin{array}{r}
\int_{0}^{\infty} \exp \left(\int_{0}^{s} \gamma_{1}(\tau) d \tau\right)\left(1+\int_{0}^{s} \gamma_{0}(\tau) d \tau\right) \times \\
\left(l_{1}(s)+l_{2}(s) \gamma_{1}(s)\right) d s<\infty .
\end{array}
$$

The above assumptions and the results from [22] imply

Theorem 1: If $\left(\mathrm{H}_{1}\right)-\left(\mathrm{H}_{7}\right)$ hold, then $V$ is bounded, lower semicontinuous on $\mathbb{R}_{+} \times E$, and $V(t, \cdot)$ is Lipschitz of rank $k(t)$ on $E$ for every $t \in \mathbb{R}_{+}$with a continuous decreasing function $k: \mathbb{R}_{+} \rightarrow \mathbb{R}_{+}$satisfying $k(t) \rightarrow 0$ as $t \rightarrow \infty$.

\section{A. Subdifferentiability of the Value Function}

Let us denote by $L_{x}^{-}(t, \bar{x}, \bar{y} ; v)\left(\right.$ resp. $\left.L_{x}^{+}(t, \bar{x}, \bar{y} ; v)\right)$ the lower (resp. upper) partial directional derivative of $L(t, \cdot, \bar{y})$ at $\bar{x} \in E$ in the direction $v \in E ; L_{y}^{-}(t, \bar{x}, \bar{y} ; v)$ and $L_{y}^{+}(t, \bar{x}, \bar{y} ; v)$ have obvious meanings. Then $\partial_{x}^{-} L(t, \bar{x}, \bar{y})$ (resp. $\partial_{x}^{+} L(t, \bar{x}, \bar{y})$ ) denotes the Dini-Hadamard partial subdifferential (resp. superdifferential) of $L(t, \cdot \bar{y})$ at $\bar{x}$; $\partial_{y}^{-} L(t, \bar{x}, \bar{y})$ and $\partial_{y}^{+} L(t, \bar{x}, \bar{y})$ have similar meanings. The Clarke partial directional derivatives $L_{x}^{\circ}(t, \bar{x}, \bar{y} ; v)$ and $L_{y}^{\circ}(t, \bar{x}, \bar{y} ; v)$, and the Clarke partial subdifferentials $\partial_{x}^{\circ} L(t, \bar{x}, \bar{y})$ and $\partial_{y}^{\circ} L(t, \bar{x}, \bar{y})$ are defined in a similar way.

We need another continuity assumption on $\Gamma$ :

$\left(\mathbf{H}_{5}^{\prime}\right) \Gamma(\cdot, x)$ is lower semicontinuous for every $x \in E$.

Using the Filippov type theorem from [22] we prove

Lemma 1: Suppose $\left(\mathrm{H}_{4}\right),\left(\mathrm{H}_{5}^{\prime}\right),\left(\mathrm{H}_{6}\right)$. Let $x_{0}(\cdot) \in \mathcal{A}_{\left(0, \xi_{0}\right)}$ and $t \in \mathbb{R}_{+}$be such that $\dot{x}_{0}(t) \in \Gamma\left(t, x_{0}(t)\right)$. Fix any $v \in \Gamma\left(t, x_{0}(t)\right)$. Then for every $h>0$ there exists $x_{h}(\cdot) \in$ $W^{1,1}([t, t+h] ; E)$ such that:

(i) $\dot{x}_{h}(s) \in \Gamma\left(s, x_{h}(s)\right)$ a.e., $x_{h}(t+h)=x_{0}(t+h)$;

(ii) $\left\|x_{h}(t)-x_{0}(t)-h\left(\dot{x}_{0}(t)-v\right)\right\|=o(h)$;

(iii) $\left\|\dot{x}_{h}(\cdot)-v\right\|_{L^{1}([t, t+h])}=o(h)$.

The results below substantially improve those from [6], [7], [9], [29].

Let $x_{0}(\cdot) \in \mathcal{A}_{\left(0, \xi_{0}\right)}$ be optimal and $[t]:=\left(t, x_{0}(t), \dot{x}_{0}(t)\right)$.

Theorem 2: Assume $\left(\mathrm{H}_{1}\right)-\left(\mathrm{H}_{4}\right),\left(\mathrm{H}_{5}^{\prime}\right),\left(\mathrm{H}_{6}\right)$ and $\left(\mathrm{H}_{7}\right)$. Then for a.e. $t \geq 0$ and any $v \in \Gamma\left(t, x_{0}(t)\right)$,

(i) $V_{x}^{-}\left(t, x_{0}(t) ; \dot{x}_{0}(t)-v\right) \leq L\left(t, x_{0}(t), v\right)-L[t]$;

(ii) $-\partial_{x}^{-} V\left(t, x_{0}(t)\right) \subset \partial_{y}^{\circ} L[t]+N_{\Gamma\left(t, x_{0}(t)\right)}\left(\dot{x}_{0}(t)\right)$.

Moreover, if for a.e. $t \geq 0, L\left(t, x_{0}(t), \cdot\right)$ is Gateaux differentiable at $\dot{x}_{0}(t)$, then for a.e. $t \geq 0$,

$-\partial_{x}^{-} V\left(t, x_{0}(t)\right) \subset\left\{\nabla_{y} L[t]\right\}+N_{\Gamma\left(t, x_{0}(t)\right)}\left(\dot{x}_{0}(t)\right)$.

Proof: (i): Let $t>0$ be such that the two inclusions in (H6) hold, $v \in \Gamma\left(t, x_{0}(t)\right)$ and $x_{h}(\cdot) \in W^{1,1}([t, t+h] ; E)$ be as in the claim of Lemma 1 . Then, for every $s \in[t, t+h]$ 
we have

$$
\begin{aligned}
\| x_{h}(s) & -x_{0}(t)\|\leq\| x_{h}(s)-x_{h}(t)\|+\| x_{h}(t)-x_{0}(t) \| \\
\leq & h\|v\|+h\left\|\dot{x}_{0}(t)-v\right\|+o(h) \\
\leq & h\left(\gamma_{0}(t)+\gamma_{1}(t)\left\|x_{h}(t)\right\|+\left\|\dot{x}_{0}(t)-v\right\|\right)+o(h) \\
\leq & h\left(\gamma_{0}(t)+\gamma_{1}(t)\left(\left\|x_{0}(t)\right\|+h\left\|\dot{x}_{0}(t)-v\right\|\right)\right. \\
& \quad+h\left\|\dot{x}_{0}(t)-v\right\|+o(h) \\
& =h\left(\gamma_{0}(t)+\gamma_{1}(t)\left\|x_{0}(t)\right\|+\left\|\dot{x}_{0}(t)-v\right\|\right)+o(h)
\end{aligned}
$$

which yields the following estimates:

$$
\begin{aligned}
& \left|\int_{t}^{t+h} L\left(s, x_{h}(s), \dot{x}_{h}(s)\right) d s-\int_{t}^{t+h} L\left(s, x_{0}(t), v\right) d s\right| \\
& \leq \int_{t}^{t+h}\left(l_{1}(s)\left\|x_{h}(s)-x_{0}(t)\right\|+l_{2}(s)\left\|\dot{x}_{h}(s)-v\right\|\right) d s \\
& \leq\left(h\left(\gamma_{0}(t)+\gamma_{1}(t)\left\|x_{0}(t)\right\|+\left\|\dot{x}_{0}(t)-v\right\|\right)+o(h)\right) \times \\
& \int_{t}^{t+h} l_{1}(s) d s+\sup _{s \in[t, t+h]} l_{2}(s)\left\|\dot{x}_{h}(\cdot)-v\right\|_{L^{1}([t, t+h])} .
\end{aligned}
$$

So the above is equal to $o(h)$. By the separability of $E$ and [24, Theorem 2.5], there exists a subset $I$ of $\mathbb{R}_{+}$such that the Lebesgue measure of its complement $\mathbb{R}_{+} \backslash I$ is zero with $\lim _{h \downarrow 0} h^{-1} \int_{t}^{t+h} L(s, x, v) d s=L(t, x, v)$ for every $(t, x, v) \in I \times E \times E$. We thus obtain: for every $t \in I$,

$$
\lim _{h \downarrow 0} \frac{1}{h} \int_{t}^{t+h} L\left(s, x_{h}(s), \dot{x}_{h}(s)\right) d s=L\left(t, x_{0}(t), v\right) .
$$

Let $t \in \mathbb{R}_{+}$be a Lebesgue point of $L\left(\cdot, x_{0}(\cdot), \dot{x}_{0}(\cdot)\right)$. By the dynamic programming,

$V\left(t, x_{h}(t)\right) \leq \int_{t}^{t+h} L\left(s, x_{h}(s), \dot{x}_{h}(s)\right) d s+V\left(t+h, x_{0}(t+h)\right)$.

Subtracting $V\left(t, x_{0}(t)\right)=\int_{t}^{t+h} L[s] d s+V\left(t+h, x_{0}(t+h)\right)$ and using that $V(t, \cdot)$ is locally Lipschitz, we get

$$
\begin{aligned}
& \int_{t}^{t+h} L\left(s, x_{h}(s), \dot{x}_{h}(s)\right) d s-\int_{t}^{t+h} L[s] d s \\
\geq & V\left(t, x_{h}(t)\right)-V\left(t, x_{0}(t)\right) \\
\geq & V\left(t, x_{0}(t)+h\left(\dot{x}_{0}(t)-v\right)\right)-V\left(t, x_{0}(t)\right)-o(h) .
\end{aligned}
$$

Dividing by $h>0$ and taking the limit as $h \rightarrow 0$ yields

$$
V_{x}^{-}\left(t, x_{0}(t) ; \dot{x}_{0}(t)-v\right) \leq L\left(t, x_{0}(t), v\right)-L[t] .
$$

Since $v \in \Gamma\left(t, x_{0}(t)\right)$ is arbitrary, we proved (i).

(ii): Let $x^{*} \in \partial_{x}^{-} V\left(t, x_{0}(t)\right), u \in K_{\Gamma\left(t, x_{0}(t)\right)}\left(\dot{x}_{0}(t)\right)$. Then for some $\theta_{n} \rightarrow 0+$ and $u_{n} \in E$ with $u_{n} \rightarrow u$ we have $\dot{x}_{0}(t)+\theta_{n} u_{n} \in \Gamma\left(t, x_{0}(t)\right)$ for each $n \in \mathbb{N}$. Since $V_{x}^{-}\left(t, x_{0}(t) ;-\theta_{n} u_{n}\right) \leq L\left(t, x_{0}(t), \dot{x}_{0}(t)+\theta_{n} u_{n}\right)-L[t]$,

$$
\left\langle x^{*},-u_{n}\right\rangle \leq \frac{L\left(t, x_{0}(t), \dot{x}_{0}(t)+\theta_{n} u_{n}\right)-L[t]}{\theta_{n}} .
$$

Letting $n \rightarrow \infty$ yields

$$
\left\langle-x^{*}, u\right\rangle \leq L_{y}^{+}\left(t, x_{0}(t), \dot{x}_{0}(t) ; u\right) \leq L_{y}^{\circ}\left(t, x_{0}(t), \dot{x}_{0}(t) ; u\right)
$$

for every $u \in K_{\Gamma\left(t, x_{0}(t)\right)}\left(\dot{x}_{0}(t)\right)$. Suppose for a moment that $-x^{*} \notin \partial_{y}^{\circ} L[t]+N_{\Gamma\left(t, x_{0}(t)\right)}\left(\dot{x}_{0}(t)\right)$. Since $\partial_{y}^{\circ} L[t]$ is weakly* compact, and $N_{\Gamma\left(t, x_{0}(t)\right)}\left(\dot{x}_{0}(t)\right)$ is weakly* closed, $\partial_{y}^{\circ} L[t]+$ $N_{\Gamma\left(t, x_{0}(t)\right)}\left(\dot{x}_{0}(t)\right)$ is weakly* closed. Then, by the separation theorem, there exists $v \in E$ such that

$$
\left\langle-x^{*}, v\right\rangle>\sup _{y^{*} \in \partial_{y}^{\circ} L[t]}\left\langle y^{*}, v\right\rangle+\sup _{z^{*} \in N_{\Gamma\left(t, x_{0}(t)\right)}\left(\dot{x}_{0}(t)\right)}\left\langle z^{*}, v\right\rangle .
$$

Thus $\left\langle z^{*}, v\right\rangle \leq 0$ for every $z^{*} \in N_{\Gamma\left(t, x_{0}(t)\right)}\left(\dot{x}_{0}(t)\right)$. This means that $v \in T_{\Gamma\left(t, x_{0}(t)\right)}\left(\dot{x}_{0}(t)\right) \subset K_{\Gamma\left(t, x_{0}(t)\right)}\left(\dot{x}_{0}(t)\right)$. The above inequality also implies that $\left.\left\langle-x^{*}, v\right\rangle\right\rangle$ $L_{y}^{\circ}\left(t, x_{0}(t), \dot{x}_{0}(t) ; v\right)$, in contradiction with (1). This proves (ii). If $t$ is so that $L\left(t, x_{0}(t), \cdot\right)$ is Gateaux differentiable and locally Lipschitz at $\dot{x}_{0}(t)$, then (1) can be replaced by

$$
\left\langle-x^{*}, u\right\rangle \leq \nabla_{y} L\left(t, x_{0}(t), \dot{x}_{0}(t) ; u\right)
$$

for every $u \in K_{\Gamma\left(t, x_{0}(t)\right)}\left(\dot{x}_{0}(t)\right)$. The above arguments are also valid when we replace $\partial_{y}^{\circ} L[t]$ and $L_{y}^{\circ}\left(t, x_{0}(t), \dot{x}_{0}(t) ; v\right)$ respectively by $\nabla_{y} L[t]$ and $\left\langle\nabla_{y} L[t], v\right\rangle$.

\section{MAXIMUM PRINCIPLE}

A function $p: \mathbb{R}_{+} \rightarrow E^{*}$ is locally absolutely continuous if its restriction to any bounded closed interval is absolutely continuous. A function $p(\cdot)$ is said to be weakly* differentiable at $t>0$ if there exists $x^{*} \in E^{*}$ such that

$$
\lim _{h \rightarrow 0}\left\langle\frac{p(t+h)-p(t)}{h}, x\right\rangle=\left\langle x^{*}, x\right\rangle .
$$

for every $x \in E$. Then $x^{*}$ is called the weak ${ }^{*}$ derivative of $p$ at $t$ and is denoted by $\dot{p}(t)$ with $\frac{d}{d t}\langle p(t), x\rangle=\langle\dot{p}(t), x\rangle$.

The weak* differentiability of locally absolutely continuous functions is fundamental in the sequel and can be deduced from the proof of [26, Lemma].

$\left(\mathbf{H}_{\mathbf{8}}\right)$ For every $t \in \mathbb{R}_{+}$there exists $\eta>0$ such that

$$
\left(x_{0}(s)+\eta B, \dot{x}_{0}(s)\right) \subset \operatorname{gph} \Gamma(s, \cdot) \quad \text { a.e. } s \in[0, t],
$$

where $\operatorname{gph} \Gamma(s, \cdot)$ stands for the graph of $\Gamma(s, \cdot)$.

Define the Hamiltonian $H: \mathbb{R}_{+} \times E \times E^{*} \rightarrow \mathbb{R} \cup\{+\infty\}$

$$
H\left(t, x, x^{*}\right):=\sup _{y \in \Gamma(t, x)}\left\{\left\langle x^{*}, y\right\rangle-L(t, x, y)\right\} .
$$

Theorem 3: Assume $\left(\mathrm{H}_{1}\right)-\left(\mathrm{H}_{4}\right),\left(\mathrm{H}_{5}^{\prime}\right),\left(\mathrm{H}_{6}\right)-\left(\mathrm{H}_{8}\right)$. If $\partial_{x}^{-} V\left(0, x_{0}(0)\right) \neq \emptyset$ and $\partial_{x}^{+} L[t]$ is nonempty a.e., then for every $x^{*} \in \partial_{x}^{-} V\left(0, x_{0}(0)\right)$ there exists a locally absolutely continuous $p: \mathbb{R}_{+} \rightarrow E^{*}$ with $p(0)=-x^{*}$ such that

(i) $-p(t) \in \partial_{x}^{-} V\left(t, x_{0}(t)\right)$ for every $t \in \mathbb{R}_{+}$;

(ii) $p(t) \in \partial_{y}^{\circ} L[t]+N_{\Gamma\left(t, x_{0}(t)\right)}\left(\dot{x}_{0}(t)\right)$ a.e.;

(iii) $\dot{p}(t) \in \partial_{x}^{+} L[t]$ a.e.;

(iv) $\left.H\left(t, x_{0}(t), p(t)\right)=\left\langle p(t), \dot{x}_{0}(t)\right\rangle-L\left(t, x_{0}(t)\right), \dot{x}_{0}(t)\right)$ a.e.;

(v) $\lim _{t \rightarrow \infty} p(t)=0$,

where $\dot{p}(t)$ denotes the weak ${ }^{*}$ derivative of $p(\cdot)$. In particular, if $\partial_{x}^{-} V\left(0, x_{0}(0)\right)$ is nonempty, then $\partial_{x}^{-} V\left(t, x_{0}(t)\right)$ is nonempty for every $t \in \mathbb{R}_{+}$.

Proof: Let $t \in \mathbb{R}_{+}$and $\eta>0$ be as in $\left(\mathrm{H}_{8}\right)$. Fix any $x^{*} \in \partial_{x}^{-} V\left(0, x_{0}(0)\right)$ and let $f:[0, t] \rightarrow E^{*}$ be a Gelfand integrable selector of the mapping $s \mapsto \partial_{x}^{+} L[s]$ over the interval $[0, t]$. Define $p(t)=\int_{0}^{t} f(s) d s-x^{*}$ as a Gelfand integral. We claim that $-p(t) \in \partial_{x}^{-} V\left(t, x_{0}(t)\right)$. To this end, fix any $v \in E$ and consider the local perturbation of $x_{0}(\cdot)$ over $[0, t]$ given by $x_{\theta}(s):=x_{0}(s)+\theta v$ for $s \in[0, t]$. By construction, $\dot{x}_{\theta}(s)=\dot{x}_{0}(s)$ a.e. $s \in[0, t]$ 
and $x_{\theta}(s) \in x_{0}(s)+\eta B$ whenever $0<\theta \leq(1+\|v\|)^{-1} \eta$, and hence, $\left(x_{\theta}(s), \dot{x}_{\theta}(s)\right) \in \operatorname{gph} \Gamma(s, \cdot)$ a.e. $s \in[0, t]$. Thus

$$
V\left(0, x_{\theta}(0)\right) \leq \int_{0}^{t} L\left(s, x_{\theta}(s), \dot{x}_{\theta}(s)\right) d s+V\left(t, x_{\theta}(t)\right) .
$$

Subtracting $V\left(0, x_{0}(0)\right)=\int_{0}^{t} L[s] d s+V\left(t, x_{0}(t)\right)$ yields

$$
\begin{gathered}
V\left(0, x_{\theta}(0)\right)-V\left(0, x_{0}(0)\right) \leq V\left(t, x_{\theta}(t)\right)-V\left(t, x_{0}(t)\right)+ \\
\int_{0}^{t}\left(L\left(s, x_{\theta}(s), \dot{x}_{\theta}(s)\right)-L[s]\right) d s .
\end{gathered}
$$

Let $\theta_{n} \rightarrow 0+$ be such that

$$
V_{x}^{-}\left(t, x_{0}(t) ; v\right)=\lim _{n \rightarrow \infty} \frac{V\left(t, x_{0}(t)+\theta_{n} v\right)-V\left(t, x_{0}(t)\right)}{\theta_{n}} .
$$

Using the Lebesgue dominated convergence theorem and Fatou's lemma and taking the limit as $n \rightarrow \infty$ yields

$$
\begin{aligned}
& V_{x}^{-}\left(0, x_{0}(0) ; v\right) \leq \int_{0}^{t} L_{x}^{+}\left(s, x_{0}(s), \dot{x}_{0}(s) ; v\right) d s+ \\
& V_{x}^{-}\left(t, x_{0}(t) ; v\right) \leq \int_{0}^{t}\langle f(s), v\rangle d s+V_{x}^{-}\left(t, x_{0}(t) ; v\right) \\
& =\langle p(t), v\rangle+\left\langle x^{*}, v\right\rangle+V_{x}^{-}\left(t, x_{0}(t) ; v\right) \quad \forall v \in E .
\end{aligned}
$$

Since $\left\langle x^{*}, v\right\rangle \leq V_{x}^{-}\left(0, x_{0}(0) ; v\right)$, we obtain $\langle-p(t), v\rangle \leq$ $V_{x}^{-}\left(t, x_{0}(t) ; v\right)$ for every $v \in E$ and (i) follows.

Since $\langle p(t), y\rangle=\int_{0}^{t}\langle f(s), y\rangle d s-\left\langle x^{*}, y\right\rangle$ for all $t \in \mathbb{R}_{+}$, $y \in E$ and $|\langle f(s), y\rangle| \leq\|f(s)\|\|y\| \leq l_{1}(s)\|y\|$, we get $|\langle p(t+h)-p(t), y\rangle| \leq\|y\| \int_{t}^{t+h} l_{1}(s) d s$, and therefore, $\|p(t+h)-p(t)\| \leq \int_{t}^{t+h} l_{1}(s) d s$ for every $h>0$. Hence $p$ is locally absolutely continuous and its weak ${ }^{*}$ derivative $\dot{p}(t)=f(t)$ exists a.e. This proves (iii). By Theorem 2 and (i), $\left\langle p(t), \dot{x}_{0}(t)\right\rangle-L[t] \geq\langle p(t), y\rangle-L\left(t, x_{0}(t), y\right)$ for every $y \in \Gamma\left(t, x_{0}(t)\right)$ implying the maximum principle (iv). Thus, for a.e. $t \in \mathbb{R}_{+}$and every $v \in T_{\Gamma\left(t, x_{0}(t)\right)}\left(\dot{x}_{0}(t)\right)$, we have $\langle p(t), v\rangle \leq L_{y}^{-}\left(t, x_{0}(t), \dot{x}_{0}(t) ; v\right) \leq L_{y}^{\circ}\left(t, x_{0}(t), \dot{x}_{0}(t) ; v\right)$ and condition (ii) follows from the separation argument as in the proof of Theorem 2. To get (v) recall that by Theorem $1, V(t, \cdot)$ is Lipschitz of rank $k(t)$ with $k(t) \rightarrow 0$ as $t \rightarrow \infty$. Therefore, $\|p(t)\| \leq k(t) \rightarrow 0$.

\section{Relaxed VARiational Problems}

The significance of Theorems 2 and 3 is severely limited whenever, $\dot{x}_{0}(t)$ happens to be an isolated point in $\Gamma\left(t, x_{0}(t)\right)$ on a set of positive measure, because on this set $N_{\Gamma\left(t, x_{0}(t)\right)}\left(\dot{x}_{0}(t)\right)$ is the whole space. To overcome this limitation, the relaxation technique provides some additional information on the subdifferentiability of the value function.

Define the multifunction $\tilde{\Gamma}: \mathbb{R}_{+} \times E \rightsquigarrow \mathbb{R} \times E$ by

$$
\tilde{\Gamma}(t, x)=\{(r, y) \in \mathbb{R} \times E \mid r=L(t, x, y), y \in \Gamma(t, x)\} .
$$

Then $\tilde{\Gamma}(t, x)$ is the augmented velocity set. The relaxed variational problem, which is a convexified problem corresponding to $\left(P_{t}\right)$, is as follows.

$$
\begin{aligned}
\tilde{V}(t, \xi)= & \inf _{\substack{\psi(\cdot) \in L^{1}\left(\mathbb{R}_{+}\right) \\
x(\cdot) \in W_{\mathrm{loc}}^{1,1}([t, \infty) ; E)}} \int_{t}^{\infty} \psi(s) d s \\
& (\psi, \dot{x})(s) \in \overline{\operatorname{co}} \tilde{\Gamma}(s, x(s)) \text { a.e., } x(t)=\xi .
\end{aligned}
$$

$\tilde{V}: \mathbb{R} \times E \rightarrow \mathbb{R} \cup\{ \pm \infty\}$ is the value function of the relaxed problem. Define the set of admissible relaxed trajectories starting at time $t \geq 0$ from the initial condition $\xi \in E$ by

$$
\begin{gathered}
\tilde{\mathcal{A}}_{(t, \xi)}:=\left\{\left(\int_{0}^{\cdot} \psi(\tau) d \tau, x(\cdot)\right) \in W_{\mathrm{loc}}^{1,1}([t, \infty) ; \mathbb{R} \times E) \mid\right. \\
(\psi(s), \dot{x}(s)) \in \overline{\operatorname{co}} \tilde{\Gamma}(s, x(s)) \text { a.e. } s \in[t, \infty), x(t)=\xi\} .
\end{gathered}
$$

For $(t, \xi) \in \operatorname{dom} \tilde{V}$, a trajectory $\left(\int_{0}^{\cdot} \psi(\tau) d \tau, x(\cdot)\right) \in \tilde{\mathcal{A}}_{(t, \xi)}$ is said to be optimal for $\left(\mathrm{RP}_{t}\right)$ if it satisfies $\int_{t}^{\infty} \psi(s) d s=$ $\tilde{V}(t, \xi)>-\infty$. Then $\tilde{V}(t, \xi) \leq V(t, \xi), \forall(t, \xi) \in \operatorname{dom} \tilde{V}$.

Denote by $\tilde{B}$ the open unit ball in $\mathbb{R} \times E$, where the norm in $\mathbb{R} \times E$ is given by $\|(r, x)\|_{\tilde{\Gamma}}:=|r|+\|x\|$. It is easy to see that if $\left(\mathrm{H}_{2}\right)-\left(\mathrm{H}_{6}\right)$ hold, then $\tilde{\Gamma}$ has nonempty closed values, is measurable with respect to the first variable and satisfies assumptions similar to $\left(H_{6}\right)$.

The following relaxation result for differential inclusions is a special case of [22, Theorem 2.5].

Lemma 2: Let $0 \leq t_{0}<t_{1}$. If $\left(\mathbf{H}_{4}\right),\left(\mathrm{H}_{5}\right)$ and $\left(\mathrm{H}_{6}\right)$ hold, then for any $y(\cdot) \in W^{1,1}\left(\left[t_{0}, t_{1}\right] ; E\right)$ with $\dot{y}(t) \in$ $\overline{\mathrm{co}} \Gamma(t, y(t))$ a.e. and every $\varepsilon>0$ there exists $x(\cdot) \in$ $W^{1,1}\left(\left[t_{0}, t_{1}\right] ; E\right)$ with $\dot{x}(t) \in \Gamma(t, x(t))$ a.e. such that $x\left(t_{0}\right)=y\left(t_{0}\right)$ and $\sup _{t \in\left[t_{0}, t_{1}\right]}\|x(t)-y(t)\|<\varepsilon$.

It implies the following theorem.

Theorem 4: If $\left(\mathrm{H}_{1}\right)-\left(\mathrm{H}_{7}\right)$ hold, then $V$ coincides with $\tilde{V}$. In particular, If $x(\cdot) \in \mathcal{A}_{(t, \xi)}$ is optimal for $\left(P_{t}\right)$, then $\left(\int_{t}^{\cdot} L(\tau, x(\tau), \dot{x}(\tau)) d \tau, x(\cdot)\right) \in \tilde{\mathcal{A}}_{(t, \xi)}$ is optimal for $\left(\mathrm{RP}_{t}\right)$.

Define the "relaxed" Lagrangian $\tilde{L}: \mathbb{R}_{+} \times E \times E \rightarrow$ $\mathbb{R} \cup\{+\infty\}$ of $L$ by: for any $y \in \overline{\operatorname{co}} \Gamma(t, x)$

$$
\tilde{L}(t, x, y)=\inf \{r \in \mathbb{R} \mid(r, y) \in \overline{\operatorname{co}} \tilde{\Gamma}(t, x)\}
$$

and set $\tilde{L}(t, x, y)=+\infty$ if $y \notin \overline{\operatorname{co}} \Gamma(t, x)$.

Then $\tilde{L} \leq L$ on gph $\overline{\operatorname{co}} \tilde{\Gamma}$, and $\tilde{L}(t, x, \cdot)$ is lower semicontinuous and convex on $E$ for every $(t, x) \in \mathbb{R}_{+}$.

$\left(\mathbf{H}_{\mathbf{2}}^{\prime}\right) L(\cdot, x, y)$ is continuous for every $(x, y) \in E \times E$.

Theorem 5: If $\left(\mathrm{H}_{1}\right),\left(\mathrm{H}_{2}^{\prime}\right),\left(\mathrm{H}_{3}\right),\left(\mathrm{H}_{4}\right),\left(\mathrm{H}_{5}^{\prime}\right),\left(\mathrm{H}_{6}\right)$ and $\left(\mathrm{H}_{7}\right)$ hold, then:

(i) $V_{x}^{-}\left(t, x_{0}(t) ; \dot{x}_{0}(t)-v\right) \leq \tilde{L}\left(t, x_{0}(t), v\right)-\tilde{L}[t]$ for a.e. $t \in \mathbb{R}_{+}$and every $v \in \overline{\mathrm{co}} \Gamma\left(t, x_{0}(t)\right)$;

(ii) $-\partial_{x}^{-} V\left(t, x_{0}(t)\right) \subset \partial_{y}^{-} \tilde{L}[t]$ a.e. $t \in \mathbb{R}_{+}$;

(iii) $\partial_{y}^{-} \tilde{L}[t] \subset \partial_{y}^{\circ} L[t]+N_{\overline{\mathrm{co}} \Gamma\left(t, x_{0}(t)\right)}\left(\dot{x}_{0}(t)\right)$ a.e. $t \in \mathbb{R}_{+}$.

Proof: (i): $\left(\mathrm{H}_{3}\right)$ implies that for a.e. $t \geq 0, \tilde{L}(t, \cdot, \cdot)$ has finite values on gph $\overline{\mathrm{co}} \Gamma(t, \cdot)$. Since $l_{2}(\cdot)$ is locally bounded, $\left(\mathrm{H}_{2}^{\prime}\right)$ guarantees that $L(\cdot, x, \cdot)$ is continuous on $\mathbb{R}_{+} \times E$ for every $x \in E$. Then $\tilde{\Gamma}(\cdot, x)$ is lower semicontinuous, and hence, $\overline{\operatorname{co}} \tilde{\Gamma}(\cdot, x)$ is also lower semicontinuous. Let $t \in \mathbb{R}_{+}$ be a Lebesgue point of $s \mapsto \int_{0}^{s} L\left(\tau, x_{0}(\tau), \dot{x}_{0}(\tau)\right) d \tau$ such that $\dot{x}_{0}(t) \in \Gamma\left(t, x_{0}(t)\right)$. Take any $(r, v) \in \overline{\mathrm{co}} \tilde{\Gamma}\left(t, x_{0}(t)\right)$. Applying Lemma 1 to $\overline{\operatorname{co}} \tilde{\Gamma}$, for every $h>0$ there exists $\left(a_{h}(\cdot), x_{h}(\cdot)\right) \in W^{1,1}([t, t+h] ; \mathbb{R} \times E)$ such that:

(a) $\left(\dot{a}_{h}, \dot{x}_{h}\right)(s) \in \overline{\mathrm{co}} \tilde{\Gamma}\left(s, x_{h}(s)\right)$ a.e., $x_{h}(t+h)=x_{0}(t+h)$;

(b) $\left\|x_{h}(t)-x_{0}(t)-h\left(\dot{x}_{0}(t)-v\right)\right\|=o(h)$;

(c) $\left\|\dot{a}_{h}(\cdot)-r\right\|_{L^{1}([t, t+h])}+\left\|\dot{x}_{h}(\cdot)-v\right\|_{L^{1}([t, t+h])}=o(h)$.

By Theorem 4, $V\left(t, x_{h}(t)\right)=$

$$
\tilde{V}\left(t, x_{h}(t)\right) \leq \int_{t}^{t+h} \dot{a}_{h}(s) d s+V\left(t+h, x_{0}(t+h)\right) .
$$


Subtracting $V\left(t, x_{0}(t)\right)=\int_{t}^{t+h} L[s] d s+V\left(t+h, x_{0}(t+h)\right)$ from the both sides of the above inequality yields

$$
\begin{array}{r}
\int_{t}^{t+h} \dot{a}_{h}(s) d s-\int_{t}^{t+h} L[s] d s \geq V\left(t, x_{h}(t)\right)-V\left(t, x_{0}(t)\right) \\
\geq V\left(t, x_{0}(t)+h\left(\dot{x}_{0}(t)-v\right)\right)-V\left(t, x_{0}(t)\right)-o(h) .
\end{array}
$$

Thus $V_{x}^{-}\left(t, x_{0}(t) ; \dot{x}_{0}(t)-v\right) \leq r-L[t]$. We proved that for every $v \in \overline{\mathrm{co}} \Gamma\left(t, x_{0}(t)\right)$

$$
V_{x}^{-}\left(t, x_{0}(t) ; \dot{x}_{0}(t)-v\right) \leq \tilde{L}\left(t, x_{0}(t), v\right)-\tilde{L}[t] .
$$

(ii): Take any $x^{*} \in \partial_{x}^{-} V\left(t, x_{0}(t)\right)$. In the same way as in the proof of Theorem 2(ii), we obtain

$$
\left\langle-x^{*}, v\right\rangle \leq \tilde{L}_{y}^{-}\left(t, x_{0}(t), \dot{x}_{0}(t) ; v\right)
$$

$\forall v \in K_{\overline{\mathrm{co}} \Gamma\left(t, x_{0}(t)\right)}\left(\dot{x}_{0}(t)\right)$. If $v \notin K_{\overline{\mathrm{co}} \Gamma\left(t, x_{0}(t)\right)}\left(\dot{x}_{0}(t)\right)$, then $\tilde{L}_{y}^{-}\left(t, x_{0}(t), \dot{x}_{0}(t) ; v\right)=+\infty$. Thus inequality (3) is true for every $v \in E$. Therefore, $-x^{*} \in \partial_{y}^{-} \tilde{L}[t]$.

(iii): If $\partial_{y}^{-} \tilde{L}[t]$ is empty, then the inclusion is trivial. Assume next that $\partial_{y}^{-} \tilde{L}[t]$ is nonempty. Observe that $\tilde{L}[t]=$ $L[t]$ a.e. $t \in \mathbb{R}_{+}$, for otherwise we must have $V\left(0, \xi_{0}\right) \neq$ $\tilde{V}\left(0, \xi_{0}\right)$, which contradicts to Theorem 4 . Since $\tilde{L} \leq L$,

$$
\tilde{L}_{y}^{-}\left(t, x_{0}(t), \dot{x}_{0}(t) ; v\right) \leq L_{y}^{\circ}\left(t, x_{0}(t), \dot{x}_{0}(t) ; v\right)
$$

for every $v \in E$. Take any $x^{*} \in \partial_{y}^{-} \tilde{L}[t]$ and assume for a moment that $x^{*} \notin \partial_{y}^{\circ} L[t]+N_{\overline{\mathrm{co}} \Gamma\left(t, x_{0}(t)\right)}\left(\dot{x}_{0}(t)\right)$. In the same way as before we derive a contradiction.

Corollary 1: Assume $\left(\mathrm{H}_{1}\right),\left(\mathrm{H}_{2}^{\prime}\right),\left(\mathrm{H}_{3}\right),\left(\mathrm{H}_{4}\right),\left(\mathrm{H}_{5}^{\prime}\right)$, $\left(\mathrm{H}_{6}\right)-\left(\mathrm{H}_{8}\right)$. If $\partial_{x}^{-} V\left(0, x_{0}(0)\right)$ is nonempty and $\partial_{x}^{+} L[t]$ is nonempty for a.e. $t \in \mathbb{R}_{+}$, then for every $x^{*} \in$ $\partial_{x}^{-} V\left(0, x_{0}(0)\right)$ there exists a locally absolutely continuous function $p: \mathbb{R}_{+} \rightarrow E^{*}$ with $p(0)=-x^{*}$ such that all conclusions of Theorem 3 hold with $\Gamma$ replaced by $\overline{c o} \Gamma$ and moreover $p(t) \in \partial_{y}^{-} \tilde{L}[t]$ a.e. In particular, if $\partial_{x}^{-} V\left(0, x_{0}(0)\right)$ is nonempty, then $\partial_{x}^{-} V\left(t, x_{0}(t)\right)$ is nonempty for every $t \in \mathbb{R}_{+}$.

\section{Vi. Necessary Conditions without the INTERIORITY ASSUMPTION}

Hypothesis $\left(\mathrm{H}_{8}\right)$ in Theorem 3 is stringent, mostly because the velocity multifunction $\Gamma$ is too general. Hence, a "structural assumption" on $x_{0}(\cdot)$ compensates this generality. If instead some "structural" assumptions are imposed on $\Gamma$, then hypothesis $\left(\mathrm{H}_{8}\right)$ can be omitted. To illustrate this observation, we consider a standard optimal control problem.

Let $X$ be a complete separable metric space, $f: \mathbb{R}_{+} \times$ $E \times X \rightarrow E$ be a velocity function, and $U: \mathbb{R}_{+} \rightsquigarrow X$ be a control multifunction. Denote by $\mathcal{M}\left(\mathbb{R}_{+}, X\right)$ the space of measurable functions on $\mathbb{R}_{+}$with values in $X$. Define the integrand $\tilde{L}: \mathbb{R}_{+} \times E \times X \rightarrow \mathbb{R}$ by $\tilde{L}(t, x, u):=$ $L(t, x, f(t, x, u))$. The optimal control problem under consideration is as follows:

$$
\begin{aligned}
& \quad \inf _{\substack{x(\cdot) \in W_{\text {loc }}^{1,1}\left(\mathbb{R}_{+}, E\right) \\
u(\cdot) \in \mathcal{M}\left(\mathbb{R}_{+}, X\right)}} \int_{0}^{\infty} \tilde{L}(t, x(t), u(t)) d t \\
& \text { s.t. } u(t) \in U(t) \text { a.e. } t \in \mathbb{R}_{+}, \\
& \quad \dot{x}(t)=f(t, x(t), u(t)) \text { a.e. } t \in \mathbb{R}_{+}, \quad x(0)=\xi .
\end{aligned}
$$

The Hamiltonian for problem $\left(\tilde{\mathrm{P}}_{0}\right)$ is given by:

$$
H\left(t, x, x^{*}\right)=\sup _{u \in U(t)}\left\{\left\langle x^{*}, f(t, x, u)\right\rangle-\tilde{L}(t, x, u)\right\} .
$$

The velocity multifunction is defined by $\Gamma(t, x):=$ $f(t, x, U(t))$. Now impose "usual" assumptions on $f$ and $U$ in order that $\Gamma$ satisfies $\left(\mathrm{H}_{1}\right)-\left(\mathrm{H}_{4}\right),\left(\mathrm{H}_{5}^{\prime}\right),\left(\mathrm{H}_{6}\right)$, and $\left(\mathrm{H}_{7}\right)$. Note that $\partial_{x}^{+} \tilde{L}(t, x, u)$ is nonempty at $(t, x, u) \in \mathbb{R}_{+} \times E \times X$ whenever so is $\partial_{x, y}^{+} L(t, x, f(t, x, u))$ and $f(t, \cdot, u)$ is locally Lipschitz and Gateaux differentiable at $x$. Denote by $\mathcal{L}(E)$ the space of bounded linear operators on $E$.

The following reasonable hypothesis is a "structural assumption" on $f$ that dispenses with $\left(\mathrm{H}_{8}\right)$.

$\left(\mathbf{H}_{\mathbf{9}}\right)(\mathrm{i}) f$ is a Carathéodory function, i.e., $f(\cdot, x, u)$ is measurable for every $(x, u) \in E \times X$ and $f(t, \cdot, \cdot)$ is continuous for every $t \in \mathbb{R}_{+}$.

(ii) For every $R>0$ and $T>0$ there exists an integrable function $k:[0, T] \rightarrow \mathbb{R}$ such that:

a) $\|f(t, x, u)\| \leq k(t)$ for every $t \in[0, T], x \in$ $R B$, and $u \in U(t)$;

b) $f(t, \cdot u)$ is Lipschitz of rank $k(t)$ on $R B$ for every $t \in[0, T]$ and $u \in U(t)$.

(iii) $f(t, \cdot, u)$ is Fréchet differentiable on $E$ for every $(t, u) \in \mathbb{R}_{+} \times X$ and the mapping $(t, x, u) \mapsto$ $\nabla_{x} f(t, x, u)$ is continuous in the uniform operator topology of $\mathcal{L}(E)$.

Conditions $\left(\mathrm{H}_{9}\right)$-(i), (ii) guarantee the existence of solutions of the integral equation

$$
x(t)=\int_{0}^{t} f(s, x(s), u(s)) d s+\xi \quad \text { for every } t \in \mathbb{R}_{+}
$$

for any control $u(\cdot) \in \mathcal{M}\left(\mathbb{R}_{+}, X\right)$. Moreover the locally absolutely continuous function $x(\cdot): \mathbb{R}_{+} \rightarrow E$ is a unique mild solution to the ordinary differential equation (ODE) in $\left(\tilde{\mathrm{P}}_{0}\right)$ ), which has the strong derivative $\dot{x}(t)$ a.e. $t \in \mathbb{R}_{+}$(in view of the separability of $E$ and the Lebesgue theorem).

Let $\left(x_{0}(\cdot), u_{0}(\cdot)\right) \in W_{\text {loc }}^{1,1}\left(\mathbb{R}_{+}, E\right) \times \mathcal{M}\left(\mathbb{R}_{+}, X\right)$ be an optimal trajectory-control pair for optimal control problem $\left(\tilde{\mathrm{P}}_{0}\right)$. Denote by $\nabla_{x} f\left(s, x_{0}(s), u_{0}(s)\right)^{*} \in \mathcal{L}\left(E^{*}\right)$ the adjoint operator of $\nabla_{x} f\left(s, x_{0}(s), u_{0}(s)\right) \in \mathcal{L}(E)$.

Theorem 6: Suppose that $\left(\mathrm{H}_{1}\right)-\left(\mathrm{H}_{4}\right), \quad\left(\mathrm{H}_{5}^{\prime}\right), \quad\left(\mathrm{H}_{6}\right)$, $\left(\mathrm{H}_{7}\right)$, and $\left(\mathrm{H}_{9}\right)$ hold with $\Gamma(t, x)=f(t, x, U(t))$. If $\partial_{x}^{-} V\left(0, x_{0}(0)\right)$ is nonempty and the mapping $\partial_{x}^{+} \tilde{L}\left(\cdot, x_{0}(\cdot), u_{0}(\cdot)\right): \mathbb{R}_{+} \rightsquigarrow E^{*}$ admits a locally Bochner integrable selector, then there exists a locally absolutely continuous function $p: \mathbb{R}_{+} \rightarrow E^{*}$ such that:

$$
\begin{gathered}
-p(t) \in \partial_{x}^{-} V\left(t, x_{0}(t)\right) \text { for every } t \in \mathbb{R}_{+} ; \\
p(t) \in \partial_{y}^{\circ} L\left(t, x_{0}(t), f\left(t, x_{0}(t), u_{0}(t)\right)\right)+ \\
N_{\Gamma\left(t, x_{0}(t)\right)}\left(f\left(t, x_{0}(t), u_{0}(t)\right)\right) \text { a.e. } t \in \mathbb{R}_{+} ; \\
-\dot{p}(t) \in \nabla_{x} f\left(t, x_{0}(t), u_{0}(t)\right)^{*} p(t)- \\
\partial_{x}^{+} \tilde{L}\left(t, x_{0}(t), u_{0}(t)\right) \text { a.e. } t \in \mathbb{R}_{+} ; \\
H\left(t, x_{0}(t), p(t)\right)=\left\langle p(t), f\left(t, x_{0}(t), u_{0}(t)\right)\right\rangle- \\
\tilde{L}\left(t, x_{0}(t), u_{0}(t)\right) \text { a.e. } t \in \mathbb{R}_{+} ; \\
\lim _{t \rightarrow \infty} p(t)=0,
\end{gathered}
$$


where $\dot{p}(t)$ denotes the strong derivative of $p(\cdot)$ at $t \in$ $\mathbb{R}_{+}$. In particular, if $\partial_{x}^{-} V\left(0, x_{0}(0)\right)$ is nonempty, then $\partial_{x}^{-} V\left(t, x_{0}(t)\right)$ is nonempty for every $t \in \mathbb{R}_{+}$.

The proof of the above theorem is quite technical and will be published elsewhere.

Remark 1: The existence of locally Bochner integrable selectors from the superdifferential mapping $t \rightsquigarrow$ $\partial_{x}^{+} \tilde{L}\left(t, x_{0}(t), u_{0}(t)\right)$ follows from $\left(\mathrm{H}_{3}\right)$ and $\left(\mathrm{H}_{9}\right)$ whenever $E^{*}$ is separable in the dual norm. For the case with nonseparable $E^{*}$, the Fréchet differentiability of the integrand $L(t, \cdot, \cdot)$ on $E \times E$ and the continuity of $(t, x, y) \mapsto\left(\nabla_{x} L(t, x, y), \nabla_{y} L(t, x, y)\right)$ in the dual norm of $E^{*} \times E^{*}$ guarantee the local Bochner integrability of $t \mapsto \nabla_{x} \tilde{L}\left(t, x_{0}(t), u_{0}(t)\right)$ in $E^{*}$ under $\left(\mathrm{H}_{3}\right)$ and $\left(\mathrm{H}_{9}\right)$. If $\partial_{x, y}^{+} L\left(t, x_{0}(t), \dot{x}_{0}(t)\right)$ is nonempty, then take any $(p, q) \in$ $\partial_{x, y}^{+} L\left(t, x_{0}(t), \dot{x}_{0}(t)\right)$ and observe that for every $v \in E$ and $u \in U(t)$, we have:

$$
\begin{aligned}
& \tilde{L}_{x}^{+}(t, x, u ; v) \leq L_{x, y}^{+}\left(t, x, f(t, x, u) ; v, \nabla_{x} f(t, x, u) v\right) \\
\leq & \langle p, v\rangle+\left\langle q, \nabla_{x} f(t, x, u) v\right\rangle=\left\langle p+\nabla_{x} f(t, x, u)^{*} q, v\right\rangle .
\end{aligned}
$$

\section{CONCLUSIONS}

It is well known that in optimal control of finite horizon problems the adjoint state can be related to generalized gradients of the value function. In the present paper we considered an infinite horizon problem with dynamics described by a differential inclusion and derived relations of subdifferential of the value function to generalized gradients of the integrand and normals to the set of admissible velocities along the optimal trajectories. Under an additional assumption we have also shown that for any optimal trajectory there exist solutions of the Euler-Lagrange inclusions satisfying in addition the Weierstrass type conditions and a sensitivity relation.

\section{REFERENCES}

[1] Araujo, A. and J. A. Scheinkman, "Maximum principle and transversality condition for concave infinite horizon economic models", $J$. Econom. Theory 30 (1983), 1-16.

[2] Askenazy, P. and C. Le Van, "A model of optimal growth strategy", J. Econom. Theory 85 (1999), 24-51.

[3] Aubin, J.- P. and F. H. Clarke, "Shadow prices and duality for a class of optimal control problems", SIAM J. Control Optim. 17 (1979), 567586.

[4] Aubin, J.- P. and H. Frankowska, Set-Valued Analysis, Birkhäuser, Boston, 1990.

[5] Barbu, V., "Convex control problems and Hamiltonian systems on infinite intervals", SIAM J. Control Optim. 16 (1978), 895-911.

[6] Benveniste, L. M. and J.A. Scheinkman, "On the differentiability of the value function in dynamic models of economics", Econometrica 47 (1979), 727-732.

[7] Benveniste, L. M. and J. A. Scheinkman, "Duality theory for dynamic optimization models of economics: The continuous time case", $J$. Econom. Theory 27 (1982), 1-19.

[8] Beyn, W.-J., T. Pampel and W. Semmer, "Dynamical optimization and Skiba sets in economic examples", Optim. Control Appl. Mech. 22 (2001), 251-280.

[9] Bonnisseau, J. M. and C. Le Van, "On the subdifferential of the value function in economic optimization problems", J. Math. Econom. 25 (1996), 55-73.
[10] Boucekkine, R., C. Camacho and G. Fabbric, "Spatial dynamics and convergence: The spatial AK model", J. Econom. Theory 148 (2013), 2719-2736.

[11] Brock, W.A. and J.A. Scheinkman, "Global asymptotic stability of optimal control systems with applications to the theory of economic growth", J. Econom. Theory 12 (1976), 164-190.

[12] Cannarsa, P. and H. Frankowska, "Value function and optimality conditions for semilinear control problems", Appl. Math. Optim. 26 (1992), 139-169.

[13] Cannarsa, P. and H. Frankowska, "Value functions, relaxation, and transversality conditions in infinite horizon optimal control", J. Math. Anal. Appl. 457 (2018), 1188-1217.

[14] Cass, D. and K. Shell, "The structure and stability of competitive dynamical systems", J. Econom. Theory 12 (1976), 31-70.

[15] Clarke, F.H., Optimization and Nonsmooth Analysis, John Wiley \& Sons, New York, 1983.

[16] Clarke, F. H. and R. B. Vinter, "Local optimality conditions and Lipschitzian solutions to the Hamilton-Jacobi equation", SIAM J. Control Optim. 21 (1983), 856-870.

[17] Clarke, F. H. and R. B. Vinter, "The relationship between the maximum principle and dynamic programming", SIAM J. Control Optim. 25 (1987), 1291-1311.

[18] Crandall, M. G., L.C. Evans and P.L. Lions, "Some properties of viscosity solutions of Hamilton-Jacobi equations", Trans. Amer. Math. Soc. 282 (1984), 487-502.

[19] Crandall, M. G. and P. L. Lions, "Viscosity solutions of HamiltonJacobi equations", Trans. Amer. Math. Soc. 277 (1983), 1-42.

[20] Davidson, R. and R. Harris, "Non-convexities in continuous-time investment theory", Rev. Econ. Stud. 48 (1981), 235-253.

[21] Frankowska, H., "Optimal trajectories associated with a solution of the contingent Hamilton-Jacobi equation”, Appl. Math. Optim. 19 (1989), 291-311.

[22] Frankowska, H., "A priori estimates for operational differential inclusions", J. Differential Equations 84 (1990), 100-128.

[23] Frankowska, H. and M. Mazzola, "On relations of the adjoint state to the value function for optimal control problems with state constraints", Nonlinear Differential Equations and Applications, 20 (2013), 361383.

[24] Frankowska, H., S. Plaskacz and T. Rzeżuchowski, "Measurable viability theorems and the Hamilton-Jacobi-Bellman equations", $J$. Differential Equations 116 (1995), 263-305.

[25] Hartl, R.F. and P. M. Kort, "History dependence without unstable steady state: A non-differentiable framework", J. Math. Econom. 39 (2003), 891-900.

[26] Kōmura, Y., "Nonlinear semi-groups in Hilbert space", J. Math. Soc. Japan 19 (1967), 493-507.

[27] Magill, M. J.P., "Pricing infinite horizon programs", J. Math. Anal. Appl. 88 (1982), 398-421.

[28] Ramsey, F. P., "A mathematical theory of saving", Economic J. 38 (1928), 543-559.

[29] Rincón-Zapatero, J. P and M.S. Santos, "Differentiability of the value function in continuous-time economic models", J. Math. Anal. Appl. 394 (2012), 305-323.

[30] Rockafellar, R. T., "Saddle points of Hamiltonian systems in convex problems of Lagrange", J. Optim. Theory Appl. 12 (1973), 367-390.

[31] Rockafellar, R. T., "Saddle points of Hamiltonian systems in convex Lagrange problems having a nonzero discount rate", J. Econom. Theory 12 (1976), 71-113.

[32] Sagara, N., "Value functions and transversality conditions for infinitehorizon optimal control problems", Set-Valued Var. Anal. 18 (2010), $1-28$.

[33] Skiba, A. K., "Optimal growth with a convex-concave production function", Econometrica 48 (1978), 527-539.

[34] Takekuma, S.-I., "Support price theorem for the continuous time model of capital accumulation", Econometrica 50 (1982), 427-442.

[35] Wagener, F. O.O., "Skiba points and heteroclinic bifurcations, with applications to the shallow lake system", J. Econom. Dynam. Control 27 (2003), 1533-1561.

[36] Ye, J. J., "Nonsmooth maximum principle for infinite-horizon problems", J. Optim. Theory Appl. 76 (1993), 485-500. 